\title{
OPTIMAL PORTFOLIOS UNDER THE THREAT OF A CRASH
}

\author{
by \\ Ralf Korn \\ Fachbereich Mathematik, Universität Kaiserslautern, Germany \\ korn@mathematik.uni-kl.de \\ Paul Wilmott \\ Wilmott Associates, London, UK \\ paul@wilmott.com
}

\begin{abstract}
:
We consider the determination of optimal portfolios under the threat of a crash. Our main assumption is that upper bounds for both the crash size and the number of crashes occurring before the time horizon are given. We make no probabilistic assumption on the crash size or the crash time distribution. The optimal strategies in the presence of a crash possibility are characterized by a balance problem between insurance against the crash and good performance in the crash-free situation. Explicit solutions for the log-utility case are given. Our main finding is that constant portfolios are no longer optimal ones.
\end{abstract}

Key words and phrases:

Optimal portfolios, crash modelling, log-utility, equilibrium strategies, worst-case scenario 


\section{Introduction}

It is well-known that the classical lognormal stock/ Black-Scholes model is not able to explain large jumps in stock prices appearing in real-world security markets. In particular, it does not contain the possibility of a crash of the stock prices. Although these crashes are rare events, they do occur in real life; the October 1987 crash being the largest in recent memory. Therefore, there is a long tradition of modelling jumps in stock prices. A seemingly obvious candidate of a class of suitable stochastic processes is that of jump-diffusion processes (see Merton (1976) for an early reference). However, jump-diffusion approaches only lead to strategies that hedge a crash situation in the mean which is no real protection against the consequences of a jump at all. In particular, an investor following such a strategy will suffer large losses during a crash. As a contrast, by implementing the strategy that we will propose an investor need not be in fear of a crash as our strategy gives him full protection in a sense that will be made more precise in the following sections.

An alternative for modelling crashes is given in Hua and Wilmott (1997). There, the underlying assumptions are that both the maximal number of crashes in a given time interval and the biggest possible size of the crashes are known in advance. Otherwise, we do not assume to know the exact time of the crashes (or even if they occur at all) and their exact sizes. In this model, a crash is characterized by the fact that all random securities traded on the market become highly correlated at the crash time and all fall together at the same time. We will base our considerations on this model.

In the current paper, modelling crashes is not our main concern. As the title indicates, we will look at optimal portfolios when the threat of a crash before the time horizon is given. Our main aim is to show that still suitable investment in stocks can be more profitable than playing safe and investing all the funds in the riskless bond. We will arrive at a typical balance problem between obtaining good worst-case bounds for the case of a crash and also a reasonable performance should no crash occur at all. Obviously, the pure bond strategy yields excellent lower bounds but a poor performance if there is no crash. On the other hand, the portfolio processes which are optimal in the classical lognormal stock model are far too risky under the threat of a crash.

In section 2 we will start by presenting the most basic case, that of a single risky security when at most one crash can occur.

Section 3 is devoted to the multi-asset case in the log-utility setting while section 4 presents some refinements and extensions. 


\section{Optimal Portfolios under the Threat of a Crash: The single stock case}

We consider a security market consisting of a riskless bond and a single risky security with prices given by

$$
\begin{array}{ll}
d P_{0}(t)=P_{0}(t) r d t, & P_{0}(0)=1 \\
d P_{1}(t)=P_{1}(t)(\mu d t+\sigma d W(t)), & P_{1}(0)=p_{1}
\end{array}
$$

where the market coefficients are constants satisfying $\mu>r$ and $\sigma \neq 0$. The "crash feature" of the stock price is now given by the possibility of a sudden fall of the stock price before the time horizon $T$. We (temporarily) only assume that this could happen at most once between now $(" t=0 ")$ and the time horizon $T$. Our knowledge of the size of the crash is subsumed in the fact that the relative, i.e. percentage, fall in the stock price lies in the interval $\left[0, k^{*}\right]$ where the constant $0<k^{*}<1$ ("the worst possible crash") is given. Otherwise we do not make any assumption on a probabilistic distribution of the exact crash time and the exact crash size. So, before and after the crash, we are in the classical lognormal stock world. (However, we will see later that our work is not limited to this situation.)

Our main aim is to find optimal portfolio processes in the following sense (for the rigorous definition of a portfolio process and the notion of a utility function as below we refer to Korn (1997)):

Let $U(x)$ be a utility function, $\pi(t)$ a portfolio process (i.e. the process of the fraction of the total wealth invested in the stock), and let $X^{\pi}(t)$ be the wealth process corresponding to the portfolio process $\pi(t)$ and the initial wealth of $x>0$. We then look for the maximal worst-case bound for the expected utility of final wealth,

$$
\sup _{\pi(.) \in A(x)} E\left(\inf _{0 \leq t \leq T, 0 \leq k \leq k^{*}} U\left(X^{\pi}(T)\right)\right)
$$

where the final wealth $X^{\pi}(T)$ in the case of a crash of size $k$ at time $t$ is given by

$$
X^{\pi}(T)=(1-\pi(t) k) \tilde{X}^{\pi}(T)
$$

with $\tilde{X}^{\pi}(t)$ the usual wealth process corresponding to $\pi$ if there is no crash. More precisely $\tilde{X}^{\pi}(t)$ is given as the unique solution to the stochastic differential equation

$$
\begin{gathered}
d \tilde{X}^{\pi}(t)=\tilde{X}^{\pi}(t)((r(t)+\pi(t)(\mu(t)-r(t))) d t+\pi(t) \sigma(t) d W(t)), \\
\tilde{X}^{\pi}(0)=x .
\end{gathered}
$$


The above representation of $X^{\pi}(T)$ clearly shows that there are two different competing effects. Of course, for obtaining a high utility from the final wealth (in the case with or without crash) it is necessary to follow a sufficiently high portfolio process (always assumed that the mean rate of stock return $\mu$ exceeds the riskless rate of $r$ ). On the other hand, a high portfolio process at the time of the crash leads to a significant decrease of the total wealth. In particular, a portfolio process exceeding $1 / k^{*}$ bears the risk of bankruptcy if a crash occurs.

Before solving the above worst-case problem, we will highlight its main features and in particular support the above remarks by looking at the following two extreme strategies in the case of the logarithmic utility function:

\section{Two extreme strategies:}

i) $\pi(t) \equiv 0$ before the crash: "Playing safe"

For this strategy (the pure bond investment) the worst-case scenario is that that no crash occurs at all. Why is this so ? Of course, a crash would do this strategy no harm, but it would give it the possibility to switch to the optimal portfolio process in the log-utility case, $\pi(t) \equiv \pi^{*}:=(\mu-r) / \sigma^{2}$ afterwards. In the no-crash scenario the pure bond strategy would lead to the following worst-case bound of

$$
W C B_{0}=E\left(\ln \left(X^{0}(T)\right)\right)=\ln (x)+r T .
$$

ii) $\pi(t) \equiv \pi^{*}:=\frac{b-r}{\sigma^{2}}$ before the crash: "Optimal investment in the crash-free world" Of course, a crash would lead to losses in this case as we have a big stock investment. The worst-case scenario is here given by a crash of maximum size $\mathrm{k}^{*}$ (independent of time), leading to the following worst-case bound of

$$
W C B_{\pi^{*}}=E\left(\ln \left(X^{\pi^{*}}(T)\right)\right)=\ln (x)+r T+1 / 2\left(\frac{b-r}{\sigma}\right)^{2} T+\ln \left(1-\pi^{*} k^{*}\right) .
$$

\section{Insights:}

- which one of the above strategies yields the better worst-case bound (mainly) depends on time to maturity

- as a consequence of the form of the above worst-case bounds one can easily infer that a constant portfolio process cannot be the optimal one (in contrast to the crashfree setting)

- strategy i) takes too few risks to be good if no crash occurs while strategy ii) is too risky to perform well if a crash occurs, thus, an optimal strategy should balance this out . 
To make the above insights more precise on a technical level, let $v_{1}(t, x)$ be the value function of the problem (before $\pi(t)$ has been chosen at time $t$ ) if we know that on $[t, T]$ at most one crash can occur. Further, let $v_{0}(t, x)$ be the value function corresponding to the optimisation problem in the usual, crash-free Black-Scholes setting. It can also be interpreted (and we will do so) as the value function of the above problem after the crash has already happened.

\section{Important Remarks:}

a) If we compare two different investment strategies with respect to their worst-case bound then we do not compare them pathwise ("scenario-wise"). We look separately at the worst-case for both strategies which then yields the worst-case bound. So typically two different strategies have two different worst-case scenarios (even if they might have the same worst-case bound).

b) As we have assumed $\mu>r$, we do not have to consider portfolio processes $\pi(t)$ that can attain negative values as long as the utility function is increasing in $\mathrm{x}$ (which we will always assume when not stated otherwise). The reason for this is that the corresponding portfolio process $\pi(t)^{+}$would yield a higher expected final utility if no crash occurs at all and that the worst-case bound given a crash occurs would only be better than that of $\pi(t)^{+}$if $\pi(t)$ would be strictly negative for all $\mathrm{t}$. But then the worst-case scenario would be the absence of a crash. And of course, then the pure bond strategy would yield a better worst-case bound than $\pi(t)$.

\section{Proposition 1}

a) An optimal portfolio process $\pi_{o p t}(t)$ for the worst-case problem has to satisfy

$$
\begin{gathered}
v_{0}\left(t, x\left(1-\pi_{o p t}(t) k *\right)\right) \geq U\left(x e^{r(T-t)}\right), \\
\pi_{\text {opt }}(T)=0 .
\end{gathered}
$$

b) We have

$$
v_{1}(t, x) \geq U\left(x e^{r(T-t)}\right) .
$$

c) In the case of log-utility $U(x)=\ln (x)$ we must have

$$
E\left(\int_{t}^{T}\left(\pi_{o p t}(s)(\mu-r)-1 / 2 \pi_{o p t}(s)^{2} \sigma^{2}\right) d s\right) \geq 0 .
$$

\section{Proof:}

a) Both assertions of a) follow from comparison of the optimal portfolio process with the pure bond investment. The first inequality says that the optimal portfolio (i.e. the one delivering the best worst-case bound) should yield a final expected 
utility at least as big as the one obtained by pure bond investment if an immediate crash (of highest size) happens.

The final condition $\pi_{\text {opt }}(T)=0$ is implied by the fact that a crash at the time horizon should have no impact. This requirement also follows from the comparison of the optimal strategy with the pure bond one.

b) is a direct consequence of a) and the fact that the best bound should always be at least as big as the pure bond bound.

c) To see assertion c), consider the effect of no crash in the log-utility case. Then the expected final utility of the optimal strategy should be no worse than that of the pure bond investment, i.e. the assertion is implied by the inequality

$$
E\left(\ln (x)+r(T-t)+\int_{t}^{T}\left(\pi(s)(\mu-r)-1 / 2 \pi(s)^{2} \sigma^{2}\right) d s\right) \geq E(\ln (x)+r(T-t))
$$

As the above assertions were all necessary but not sufficient conditions for the existence of an optimal strategy, we will at least in the log-utility case show that there exist explicit examples of strategies that perform better than the pure bond investment even under the threat of a crash. The key for constructing such a strategy lies in (the proof) of the above assertions a) and b).

\section{Corollary 2:}

Assume $U(x)=\ln (x)$. Then we have:

a) There exist strategies $\pi($.$) with a strictly higher worst-case bound than the pure$ bond strategy.

b) There exists a strategy $\hat{\pi}($.$) such that the corresponding expected log-utility after$ an immediate crash equals the expected log-utility given no crash occurs if there exists a solution $\hat{\pi}($.$) to the differential equation$

$$
\dot{\pi}(t)=\frac{1}{k^{*}}\left(1-\pi(t) k *\left(\pi(t)(\mu-r)-1 / 2\left(\pi(t)^{2} \sigma^{2}+\left(\frac{\mu-r}{\sigma}\right)^{2}\right)\right)\right.
$$

with

$$
0 \leq \hat{\pi}(.)<\frac{1}{k^{*}} .
$$

c) If there exists an optimal portfolio process for the worst-case problem then it is a non-constant one (in contrast to the problem without the threat of a crash). 


\section{Proof:}

a) Let

$$
a(t):=\frac{1}{k *}\left[1-e^{-1 / 2\left(\frac{\mu-r}{\sigma}\right)^{2}(T-t)}\right] \wedge \frac{\mu-r}{\sigma^{2}} .
$$

We then choose $\pi(t):=\frac{a(t)}{2}$ and thus obtain

$$
E\left(\int_{t}^{T}\left(\pi(s)(\mu-r)-1 / 2 \pi(s)^{2} \sigma^{2}\right) d s\right)>0
$$

(due to $\mu>r$ ) while the explicit form of $v_{0}(t, x)$,

$$
v_{0}(t, x)=\ln (x)+\left(r+1 / 2\left(\frac{\mu-r}{\sigma}\right)^{2}\right)(T-t),
$$

yields

$$
v_{0}\left(t, x\left(1-\pi(t) k^{*}\right)\right)>U\left(x e^{r(T-t)}\right) .
$$

Hence, if there is no crash at all $\pi($.$) yields a higher final log-utility than the pure$ bond strategy and in the case of an immediate crash (at all time instants) the worstcase bound $\pi($.$) still exceeds the one of the pure bond strategy.$

b) The above requirement on $\hat{\pi}($.$) translates to the integral equation$

$$
\ln (1-\pi(t) k *)=E\left(\int_{t}^{T}\left(\pi(s)(\mu-r)-1 / 2 \pi(s)^{2} \sigma^{2}\right) d s\right)-\frac{1}{2}\left(\frac{\mu-r}{\sigma}\right)^{2}(T-t) .
$$

If now there exists a solution $\hat{\pi}($.$) to the differential equation$

$$
\dot{\pi}(t)=\frac{1}{k^{*}}(1-\pi(t) k *)\left(\pi(t)(\mu-r)-1 / 2\left(\pi(t)^{2} \sigma^{2}+\left(\frac{\mu-r}{\sigma}\right)^{2}\right)\right)
$$

(also satisfying the additional side constraint in b)) then the deterministic strategy $\hat{\pi}($.) obviously solves the above integral equation.

c) is a direct consequence of Proposition 1 a) and part a) of the corollary proved above.

\section{Remark:}

If we draw the right conclusions out of the above proposition and corollary then the solution of our problem is nearly obvious. Again, look at the two extreme strategies that we considered at beginning of this section. The pure bond strategy is too safe. 
As part a) above shows one can in fact increase the risk by investing in the stock and still be better off than with the pure bond investment. So in this case the risky position can be increased so long until the crash scenario and the no crash scenario both lead to the same worst-case bound. The situation is similar for the optimal constant portfolio of the crash-free model. Here, the risk of a high stock position should be reduced until again the crash scenario and the no crash scenario both lead to the same worst-case bound. In this sense there is a balance problem between total hedging against immediate crashes and taking full risk for obtaining a high expected final log-utility (and hoping that no crash will occur) which should be taken into account to solve the worst-case problem. As a consequence of these considerations we now look at the strategy for which the worst-case bound is attained for both an immediate crash and by the final expected log-utility if no crash occurs at all.

Theorem 3 "Best portfolio under the threat of a crash"

In the log-utility case, the portfolio process $\hat{\pi}($.$) such that the corresponding$ expected log-utility after an immediate crash equals the expected log-utility given no crash occurs which is given as the solution $\hat{\pi}($.$) of the differential equation$

$$
\dot{\pi}(t)=\frac{1}{k^{*}}(1-\pi(t) k *)\left(\pi(t)(\mu-r)-1 / 2\left(\pi(t)^{2} \sigma^{2}+\left(\frac{\mu-r}{\sigma}\right)^{2}\right)\right)
$$

and satisfies

$$
0 \leq \hat{\pi}(.)<\frac{1}{k^{*}}
$$

is an optimal portfolio process for the worst-case problem.

\section{Proof:}

i) By the explicit form of

$$
E\left(\ln \left(\tilde{X}^{\pi}(T)\right)\right)=\ln (x)+r T+E\left(\int_{0}^{T}\left(\pi(t)(\mu-r)-1 / 2 \pi(t)^{2} \sigma^{2}\right) d t\right),
$$

a portfolio process $\pi($.$) with a higher final expected log-utility than \hat{\pi}($.$) if no crash$ occurs at all has to satisfy

$$
E(\pi(t))>\hat{\pi}(t)
$$

for some t. Let $\hat{v}(t, x)$ denote the expected log-utility of terminal wealth from following the portfolio process $\hat{\pi}($.$) . Then, due to$ 


$$
\begin{aligned}
& v_{0}(t, \hat{X}(t)(1-\hat{\pi}(t) k *))=\hat{v}(t, \hat{X}(t))=\hat{v}(0, x)+\int_{0}^{t} \hat{v}_{x}(s, \hat{X}(s)) \hat{\pi}(s) \sigma d W(s) \\
& +\int_{0}^{t}\left(\hat{v}_{t}(s, \hat{X}(s))+\hat{v}_{x}(s, \hat{X}(s))(r+\hat{\pi}(s)(\mu-r))+1 / 2 \hat{v}_{x x}(s, \hat{X}(s)) \hat{\pi}(s)^{2} \sigma^{2}\right) d s,
\end{aligned}
$$

we have exactly the same worst-case bounds for all possible future times of the crash,

$$
E\left(v_{0}(t, \hat{X}(t)(1-\hat{\pi}(t) k *))\right)=E(\hat{v}(t, \hat{X}(t)))=\hat{v}(0, x)=E(\hat{v}(T, \hat{X}(T))),
$$

for the portfolio process $\hat{\pi}($.). If we would now have $\pi(0)>\hat{\pi}(0)$ then due to the construction of $\hat{\pi}($.$) the strategy \pi($.) would have a strictly lower worst-case bound. To see this note that an immediate crash would lead to a strictly smaller wealth than that corresponding to $\hat{\pi}($.). Of course if the two portfolio processes coincide at the initial time than again due to the construction of $\hat{\pi}($.$) the worst-case bound of \pi($.) cannot exceed the one for $\hat{\pi}($.). Thus, we may assume $\pi(0)<\hat{\pi}(0)$. But due to this assumption and to $(*)$ there exists a first time $t \in[0, T]$ with

$$
E(\pi(t))>\hat{\pi}(t) \text { and } E\left(\ln \left(X^{\pi}(t)\right)\right) \leq E(\ln (\hat{X}(t))) .
$$

However, at exactly that time we have

$$
E(\ln (1-\pi(t) k *)) \leq \ln \left(1-k^{*} E(\pi(t))\right)<\ln (1-\hat{\pi}(t) k *)
$$

which together with $(* *)$ and the explicit form of $v_{0}(t, x)$ lead to

$$
E\left(v_{0}\left(t, X^{\pi}(t)\left(1-\pi(t) k^{*}\right)\right)\right)<E\left(v_{0}\left(t, \hat{X}(t)\left(1-\hat{\pi}(t) k^{*}\right)\right)\right) .
$$

So, again due to the construction of $\hat{\pi}($.$) the worst-case bound of \pi($.$) cannot$ exceed the one for $\hat{\pi}($.$) . Thus, a strategy \pi($.$) with a higher final expected log-$ utility as above cannot be an optimal one for the worst-case problem.

ii) By i) a portfolio process $\pi($.) with a higher final expected log-utility than $\hat{\pi}($.) cannot have a higher worst-case bound than $\hat{\pi}($.$) . On the other hand, due to the$ construction of $\hat{\pi}($.$) , a portfolio process \pi($.) leading to a smaller final expected $\log$-utility than $\hat{\pi}($.$) automatically has a smaller worst-case bound than \hat{\pi}($.$) .$

Putting i) and ii) together yields the assertion of the above proposition.

Remark: "Uniqueness of the optimal strategy"

Due to the above theorem there only exists one (deterministic) equilibrium strategy. The main reason for this is the fact that we have only one risky stock in our market model. It is exactly the one-dimensionality that allows us to conclude the relation 
(*) in the above proof. To obtain the analogous result in the multi-stock setting we have to put in more information as there we typically have more than one equilibrium strategy. So we have the additional problem of figuring out the best such one (see section 3 ).

Example 4: Log-utility ("Maximizing growth rate")

We will from now on specify to the use of the log-utility function which can also be expressed as maximizing the growth rate of the wealth process. As implied by Theorem 3 above we obtain the optimal portfolio process in this setting via solving the corresponding differential equation. By separation of variables, we arrive at the following non-linear equation for $\hat{\pi}(t)$ :

with

$$
C-1 / 2 \frac{\sigma^{2}}{k^{*}} t=\alpha \ln \left(\frac{|\hat{\pi}(t)-\pi *|}{\left|1-\hat{\pi}(t) k^{*}\right|}\right)+\frac{\beta \hat{\pi}(t)}{\hat{\pi}(t)-\pi *}
$$

$$
C:=\alpha \ln (|\pi *|)+1 / 2 \frac{\sigma^{2} T}{k^{*}}, \quad \alpha:=\frac{k^{*}}{\left(1-\pi^{*} k^{*}\right)^{2}}, \quad \beta:=-\frac{1}{\left(1-\pi^{*} k^{*}\right) \pi^{*}}, \quad \pi^{*}:=\frac{\mu-r}{\sigma^{2}} .
$$

Uniqueness and existence of the solution of the above non-linear equation for $\hat{\pi}(t)$ can always be ensured. To see this note that for $\hat{\pi}(t)=0$ the left hand side is always bigger than the right hand one. Also the derivative of the right hand side with respect to $\hat{\pi}(t)$ is strictly positive. In the case of $\pi^{*} \leq 1 / k^{*}$ we have a pole at $\hat{\pi}(t)=\pi *$ where the right hand side equals $+\infty$. Thus, there must be a unique value for $\hat{\pi}(t)$ such that the right hand side attains the value of the left hand one. In the case of $\pi^{*}>1 / k^{*}$ a similar argument (but now with a pole at $\hat{\pi}(t)=1 / k^{*}$ ) yields the existence and uniqueness assertion.

To highlight the behaviour and the performance of the "equilibrium strategy" $\hat{\pi}(t)$ we also compute the best constant portfolio strategy in the crash setting .

Proposition 5 "Best constant portfolio under the threat of a crash"

In the log-utility case the best constant portfolio strategy for our worst-case problem is given by

$$
\tilde{\pi}=\left(1 / 2\left(\frac{\mu-r}{\sigma^{2}}+\frac{1}{k^{*}}\right)-\sqrt{1 / 4\left(\frac{\mu-r}{\sigma^{2}}-\frac{1}{k^{*}}\right)^{2}+\frac{1}{\sigma^{2} T}}\right)^{+} .
$$

In particular, for $\mu>r$ we have

$$
\tilde{\pi} \stackrel{T \rightarrow \infty}{\longrightarrow} \pi^{*} \text { in case of } \pi^{*} \leq k^{*}
$$

and 


$$
\tilde{\pi} \stackrel{T \rightarrow \infty}{\longrightarrow} k^{*} \text { in case of } \pi^{*}>k^{*} \text {. }
$$

\section{Remark:}

The above limiting results deserve a closer look: if the time horizon is big and the optimal investment in the crash free model does not lead to the possibility of a negative wealth in the crash setting then it is close to the best constant portfolio under the threat of a crash. If it bears the possibility of a negative wealth after a crash (i.e. if we are in the case of $\pi^{*}>k^{*}$ ) then with a growing horizon the investor approaches the highest possible risk of a portfolio, i.e. attaining a value close to $k^{*}$. More precisely, he takes the risk of big crash losses for attaining a high growth rate of his holdings.

Proof (of Proposition 5):

First note that if an investor follows a constant portfolio strategy then the worst time for a crash (of course of maximum size $k^{*}$ ) is just before the time horizon. To see this note that after a crash the investor is able to switch to the best possible constant portfolio strategy, $\pi *$. Thus the earlier the crash happens, the longer the investor can take advantage of investing according to $\pi *$. Given that the crash now happens immediately before the time horizon $T$ the expected log-utility of an investor using the constant portfolio process $\pi$ is given by

$$
E\left(\ln \left(X^{\pi}(T)\right)\right)=\ln (x)+r T+\left(\pi(\mu-r)-1 / 2 \pi^{2} \sigma^{2}\right) T+\ln (1-\pi k *) .
$$

Differentiating the right hand side of this expression with respect to $\pi$ and setting the derivative equal to zero (note that as a function of $\pi$ the right hand side is concave) yields

$$
\tilde{\pi}=1 / 2\left(\frac{\mu-r}{\sigma^{2}}+\frac{1}{k^{*}}\right)-\sqrt{1 / 4\left(\frac{\mu-r}{\sigma^{2}}-\frac{1}{k^{*}}\right)^{2}+\frac{1}{\sigma^{2} T}}
$$

as the only zero of the derivative which is smaller than $\min \left(1 / k^{*}, \frac{\mu-r}{\sigma^{2}}\right)$. But this value can only yield the active worst-case bound if it is non-negative. Otherwise, the no crash case would deliver the worst-case bound, a case where the pure bond investment has the best worst-case bound under all non-positive portfolio strategies. But it is easy to see that we have

$$
\tilde{\pi}>0 \Leftrightarrow \mu-r>\frac{k^{*}}{T} .
$$

Finally, the remaining convergence assertions for $T \rightarrow \infty$ follows from the explicit form of $\tilde{\pi}$. 


\section{Numerical Examples}

For the following examples we solved the above non-linear equation for $\hat{\pi}(t)$ numerically (of course under the additional requirement of $0 \leq \hat{\pi}(t)<1 / k^{*}$ ). Figure 1 shows $\hat{\pi}(t)$ as a function of time for the choice of $\mu=0,2, r=0,05, \sigma=0,4, k^{*}=$ 0,2 and $T=1$. Note that even at the initial time $\mathrm{t}=0$ the optimal portfolio process in view of a crash is considerably below the optimal portfolio $\pi^{*}$ in the "crash-free" standard model which is $\pi^{*}=0,9375$. However, even the small values of the crash optimal portfolio process are much bigger than that of the best constant portfolio process in the crash setting which equals zero. These small values can be explained by the fact that if the time horizon is close then the crash risk dominates the possibilities of obtaining a better return via stock investment.

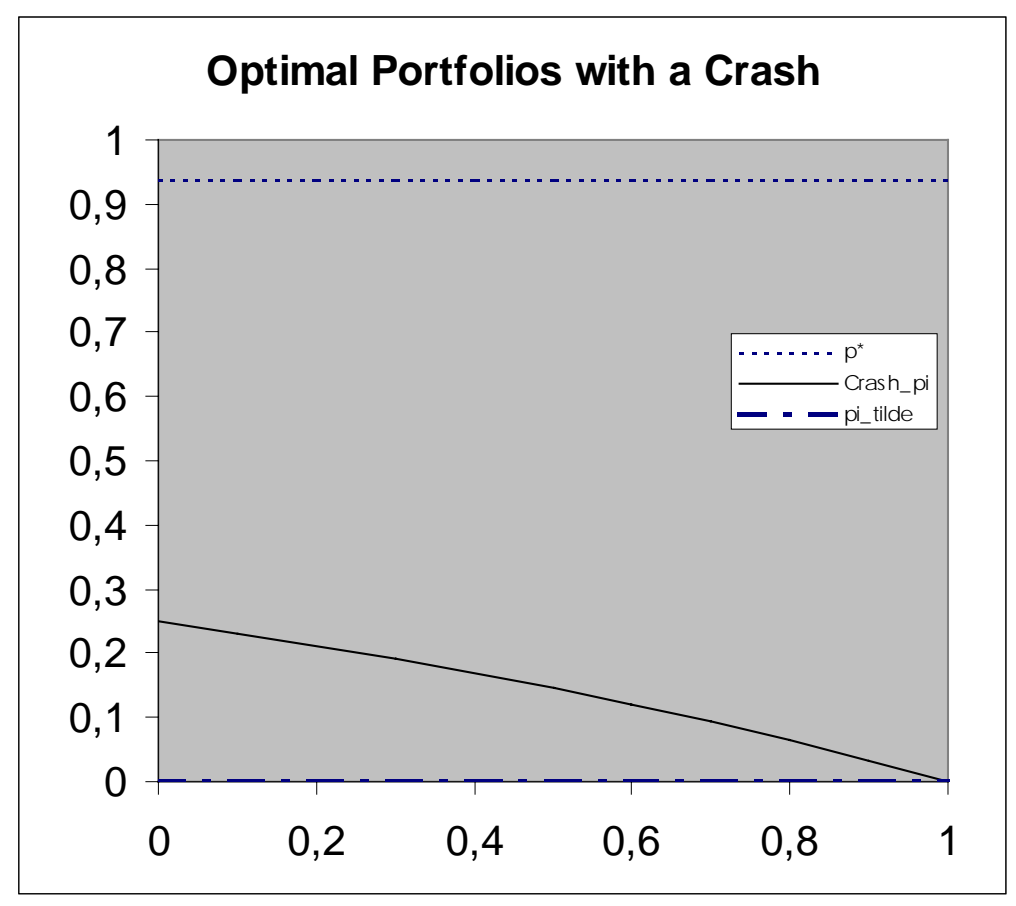

Figure 1: Optimal portfolios with and without crash possibility (small time horizon)

If, however, the time horizon is far away then the picture changes significantly. For the same data but now with $T=10$ the resulting optimal portfolio processes are given in Figure 2. Now the optimal crash portfolio is much higher (at least at times much smaller than 10) and the optimal constant portfolio in the crash setting even exceeds it. The interpretation of this behaviour is obvious. The longer the time to the trading horizon the more attractive it is to invest in the stock, and even a 
"moderate crash" is no real threat. If however the final time is near then it is good to save the gains (i.e. reduce stock investment) as then there is not enough time to compensate the effect of a crash via an optimal stock investment afterwards.

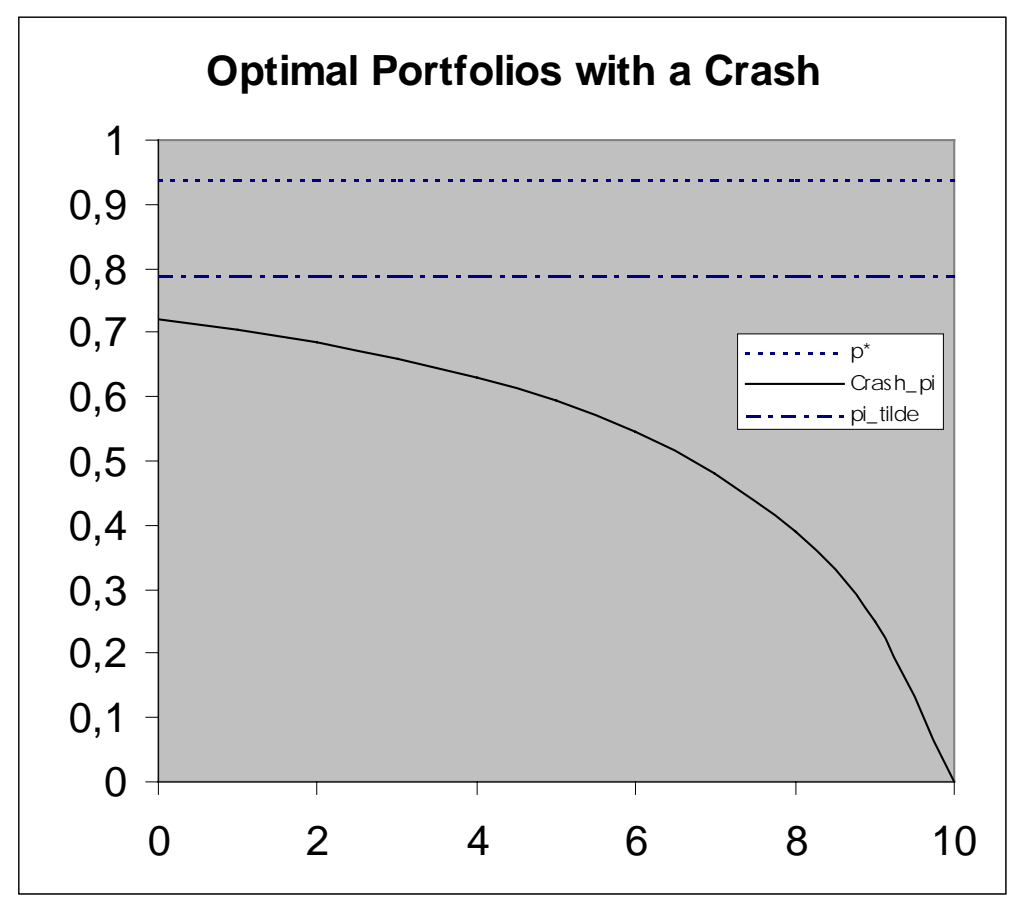

Figure 2: Optimal portfolios with and without crash possibility (large time horizon)

\section{Maximizing Growth Rate under the Threat of a Crash: n stocks}

We are now considering a market that consists of one riskless bond and $\mathrm{n}$ stocks. The prices of the stocks are assumed to follow geometric Brownian motions in "normal" times, i.e. they are given by

$$
\begin{array}{ll}
d P_{0}(t)=P_{0}(t) r d t, & P_{0}(0)=1 \\
d P_{i}(t)=P_{i}(t)\left(\mu_{i} d t+\sum_{j=1}^{n} \sigma_{i j} d W_{j}(t)\right), & P_{i}(0)=p_{i}, \quad i=1, \ldots, n
\end{array}
$$

as long as there is no crash. At the time of a crash we take on the view of Hua and Wilmott (1997) who assume that all stock prices become highly correlated and all fall at the same time as a certain index. The absolute values of all these falls are then given as suitable multiples $k_{i}$ (the so-called crash coefficients) of the percentage jump of the index. As in the one-stock case we assume that there occurs 
at most one crash and that the crash sizes in the assets are in the intervals $\left[0, k_{i}\right]$. So for simplicity we assume that the jump in the index lies in the unit interval.

As in the closing part of the preceding section we here restrict ourselves to the use of the log-utility function. The main difference to the one-stock setting is that now there can exist more than one equilibrium strategy (i.e. portfolio processes with a worst-case bound which is determined simultaneously by all future time points and events). To see this note that one can obtain equilibrium strategies by simply restricting to the sub markets made up of the bond and one arbitrary of the $n$ stocks. However, it is then natural to conjecture that the best equilibrium strategy (i.e. the one delivering the highest worst-case bound) solves our worst-case bound portfolio problem. This will indeed be the contents of the following

\section{Theorem 6}

Assume that we are in the market setting as given above. Then the optimal portfolio process is given as the deterministic portfolio process which has the highest worstcase bound under all deterministic portfolio processes satisfying the "equilibrium condition"

$$
v_{0}\left(t, x\left(1-\pi(t)^{\prime} k *\right)\right)=v_{\pi}(t, x) .
$$

Here, $v_{0}(t, x)$ is the value function in the crash free setting and $v_{\pi}(t, x)$ denotes the expected log-utility of terminal wealth from following the portfolio process $\pi($.$) . I.e. the above optimal strategy is determined as the solution of the problem$

$$
\begin{aligned}
& \sup _{\pi_{\mid[t, T]}\left(v_{\pi}(t, x)-v_{0}\left(t, x\left(1-\pi(t)^{\prime} k^{*}\right)\right)\right)=0} \\
& (* *)
\end{aligned}
$$

where the supremum is only taken over all such deterministic portfolio processes on $[t, T]$ that satisfy the equilibrium constraint $(*)$.

\section{Proof:}

To see the above claim we collect some facts:

i) Let $f(\pi):=\ln \left(1-\pi^{\prime} k^{*}\right), \pi \in \prod_{i=1}^{n}\left[0,1 / k_{i}^{*}\left[, \pi^{\prime} k^{*}<1\right.\right.$. Then $f(\pi)$ is concave for all such admissible vectors $\pi$. Hence, for each admissible portfolio process $\pi(t)$ we obtain

$$
E\left(\ln \left(1-\pi(t)^{\prime} k^{*}\right)\right) \leq \ln \left(1-(E(\pi(t)))^{\prime} k *\right)
$$


Also, it is easy to see that we can again restrict ourselves to portfolio vectors $\pi$ having non-negative components as an optimal portfolio process in the sense of our worst-case problem has to be non-negative (at least $P \otimes l-a . s$.).

ii) Let $h(\pi):=\pi{ }^{\prime}(\mu-r \underline{1})-1 / 2 \pi{ }^{\prime} \sigma \sigma^{\prime} \pi$. This function is also concave yielding

$$
E\left(\pi(t){ }^{\prime}(\mu-r \underline{1})-1 / 2 \pi(t){ }^{\prime} \sigma \sigma^{\prime} \pi(t)\right) \leq(E(\pi(t))){ }^{\prime}(\mu-r \underline{1})-1 / 2(E(\pi(t))){ }^{\prime} \sigma \sigma^{\prime}(E(\pi(t)))
$$

iii) As in the one-dimensional case it can now be shown that every portfolio process $\hat{\pi}($.$) which satisfies the equilibrium condition (*) also satisfies$

$$
E\left(v_{0}\left(t, \hat{X}(t)\left(1-\hat{\pi}(t)^{\prime} k *\right)\right)\right)=E(\hat{v}(t, \hat{X}(t)))=\hat{v}(0, x)=E(\hat{v}(T, \hat{X}(T)))
$$

where $\hat{v}(t, \hat{X}(t))$ denotes the expected log-utility of terminal wealth from following the portfolio process $\hat{\pi}($.$) .$

iv) By the explicit form of

$$
E\left(\ln \left(\tilde{X}^{\pi}(T)\right)\right)=\ln (x)+r T+E\left(\int_{0}^{T}\left(\pi(t)^{\prime}(\mu-r)-1 / 2 \pi(t)^{\prime} \sigma \sigma^{\prime} \pi(t)\right) d t\right),
$$

a portfolio process $\pi($.$) with a higher final expected log-utility than a deterministic$ "equilibrium process" $\hat{\pi}($.$) if no crash occurs at all has to satisfy$

$$
\left.\hat{\pi}(t)^{\prime}(\mu-r \underline{1})-1 / 2 \hat{\pi}(t)^{\prime} \sigma \sigma^{\prime} \hat{\pi}(t) \leq E(\pi(t)){ }^{\prime}(\mu-r \underline{1})-1 / 2(E(\pi(t)))\right)^{\prime} \sigma \sigma^{\prime}(E(\pi(t)))
$$

for some $t$.

v) Let us now prove optimality of the equilibrium portfolio process $\hat{\pi}($.$) that admits$ the highest expected log-utility of terminal wealth $\hat{v}(t, x)$ in the crash free situation under all deterministic equilibrium strategies. Therefore, consider a portfolio process $\pi($.$) with a higher final expected log-utility than \hat{\pi}($.$) . Such a process can only$ yield a higher worst-case bound than $\hat{\pi}($.$) if it also satisfies$

$$
\hat{\pi}(0)^{\prime} k^{*}>\pi(0)^{\prime} k^{*} \text {. }
$$

vi) Due to the definition of $\hat{\pi}(t)$ it attains the minimum value of $\pi^{\prime} k^{*}$ among all those vectors $\pi$ that are at the same level set of $h(\pi)$ as $\hat{\pi}(t)$ (at least for almost all $t \in[0, T]$, because otherwise one can construct a better deterministic equilibrium strategy). Consequently, as long as we have

$$
\hat{\pi}(t)^{\prime} k^{*}>(E(\pi(t)))^{\prime} k^{*}
$$

we also have

$$
E\left(\ln \left(X^{\pi}(t)\right)\right) \leq E(\ln (\hat{X}(t))) .
$$


However, due to iv) there must be a first time $t$ where we still have the above inequality between the expected log-wealth but also

$$
\hat{\pi}(t)^{\prime}(\mu-r \underline{1})-1 / 2 \hat{\pi}(t)^{\prime} \sigma \sigma^{\prime} \hat{\pi}(t) \leq E(\pi(t)) '(\mu-r \underline{1})-1 / 2(E(\pi(t))){ }^{\prime} \sigma \sigma^{\prime}(E(\pi(t))) .
$$

But due to those two relations and to ii) we then have

$$
E\left(v_{0}\left(t, X^{\pi}(t)\left(1-\pi(t)^{\prime} k^{*}\right)\right)\right)<E\left(v_{0}\left(t, \hat{X}(t)\left(1-\hat{\pi}(t)^{\prime} k^{*}\right)\right)\right)=\hat{v}(0, x)
$$

which proves optimality of $\hat{\pi}(t)$.

\section{Remark:}

At first sight the optimisation problem (**) seems to be very hard to solve. However, as by the explicit forms of both $v_{0}(t, x)$ and $v_{\pi}(t, x)$ the function over which the supremum is taken does not depend on the underlying stochastic process $X^{\pi}(t)$ one is at least able to get a numerical solution via backwards induction starting with $\pi(T)=0$.

\section{Maximizing Growth Rate under the Threat of a Crash: an arbitrary number of crashes and other refinements}

i) Arbitrary upper bound for the number of crashes

So far the maximum number of crashes was limited to one. However the extension to an arbitrary (but fixed) upper bound is straight forward. In fact the extension is something like a backward induction principle. If one has determined the best strategy given the maximum number of crashes is $n-1$ then one can imitate the above proof of Theorem 3 (or Theorem 6, respectively) to get the optimal strategy in the case of the upper bound of $n$. Simply note that the role of $v_{0}(t, x)$ is then taken over by $v_{n-1}(t, x)$, the value function for the $n-1$ case. For completeness we give the corresponding theorem in the one stock case. The $n$ stock case is then similar to Theorem 6 but lacks the explicit formula that we can give in the single stock case:

Theorem 7 "Best portfolio under the threat of at most $n$ crashes"

The optimal strategy in the log-utility case if at most $n$ crashes of size $k \in[0, k *]$ can occur is given by the unique solution(s) of the differential equation(s)

$$
\begin{gathered}
\dot{\hat{\pi}}_{j}(t)=\frac{1}{k^{*}}\left(1-\hat{\pi}_{j}(t) k *\right)\left(\hat{\pi}_{j}(t)(\mu-r)-1 / 2\left(\hat{\pi}_{j}(t)^{2} \sigma^{2}+\hat{\pi}_{j-1}(t)^{2} \sigma^{2}\right)\right) \\
\hat{\pi}_{j}(T)=0
\end{gathered}
$$

with 


$$
0 \leq \hat{\pi}_{j}(.)<\frac{1}{k^{*}}, \quad j=1, \ldots, n, \hat{\pi}_{0}(t)=\frac{\mu-r}{\sigma^{2}}
$$

where $\hat{\pi}_{j}($.$) is the optimal portfolio process for the worst-case problem if at most j$ crashes can occur.

\section{Proof:}

We give an induction on $n$, the maximum number of crashes. For the case of $n=1$ (and also $n=0$ ) all the claims follow from Theorem 3. Now that the above assertions are already proved for $n-1$ and that $\hat{\pi}_{n-1}($.$) is the corresponding equilibrium$ strategy (i.e. all future time instant yield binding constraints simultaneously for the worst case problem). Then, by induction, $v_{n-1}(t, x)$ the corresponding value function is given by

$$
v_{n-1}(t, x)=\ln (x)+r(T-t)+\ln \left(1-\hat{\pi}_{n-1}(t) k *\right)+\int_{t}^{T}\left((\mu-r) \hat{\pi}_{n-2}(s)-1 / 2 \hat{\pi}_{n-2}(s)^{2} \sigma^{2}\right) d s
$$

(where this only holds for $n-1>0$ ). The rest of the proof is now totally similar to that of Theorem 3 with an obvious change of notation.

We illustrate the n-crash-situation by the following figure which shoes the situation for the 3-crash-situation with the same data as those which have been used for producing Figure 2. Of course, the more crashes possibly to come the less is the optimally invested fraction of wealth into the risky stock. 


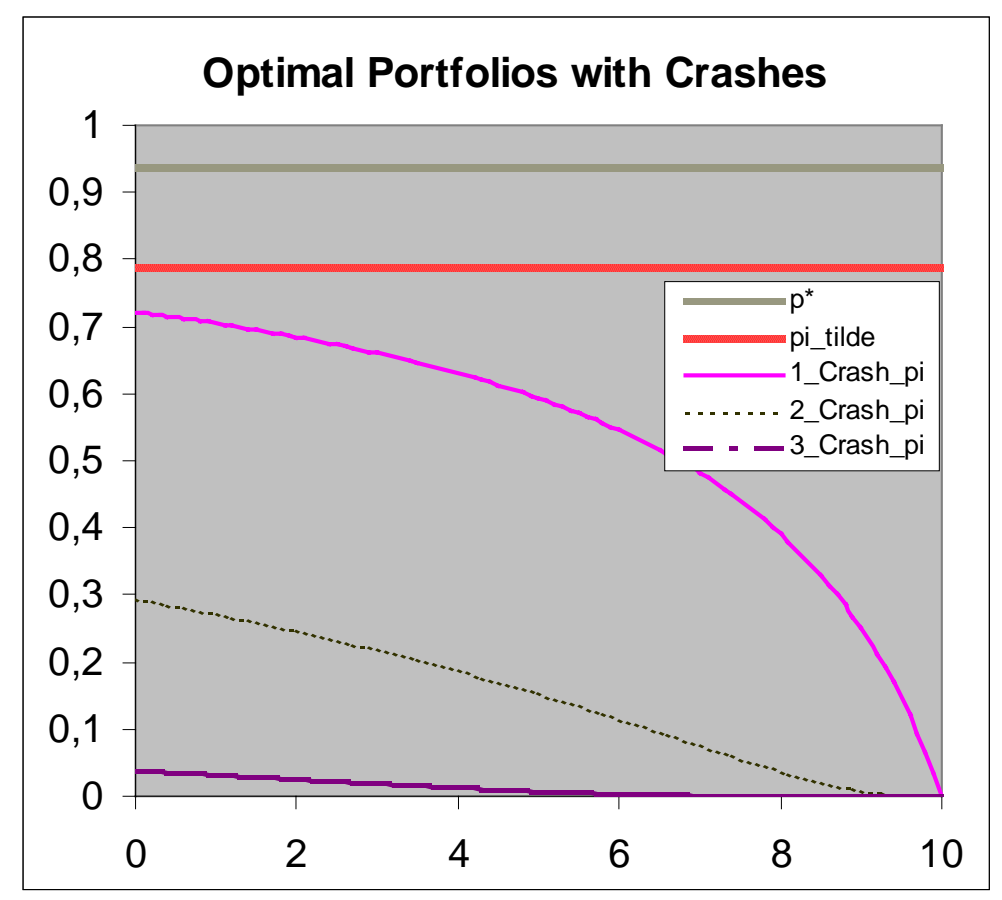

Figure 3: Optimal portfolios with and without crash possibility (large time horizon, at most three crashes)

ii) Changing volatility after a crash

It is a common phenomenon that after a crash has happened the volatility has the tendency to increase. For our worst-case problem this has the consequence that the "starting value function" $v_{0}(t, x)$ has to be computed with a different value of $\sigma$. In particular, in the $n$ crash case it might be necessary to calculate all value functions with different values of $\sigma$ where they are valid.

iii) Further possible refinements

There are still a lot of possible problems in the above setting which are worth to consider and which might be subject of future research:

- inclusion of the possibility for consumption

- explicit solution of the problem for a general utility function

- inclusion of liquidity constraints

- additional consideration of derivatives for portfolio insurance 


\section{References:}

Black, F \& Scholes, M 1973 The pricing of options and corporate liabilities.

Journal of Political Economy 81 637-59

Hua, P \& Wilmott, P 1997 Crash courses. Risk magazine 10 (6) 64-67 (June)

Korn, R 1997 Optimal Portfolios. World Scientific

Merton, RC 1976 Option pricing when underlying stock returns are discontinuous.

Journal of Financial Economics 3 125-44 